\title{
Methodologies for localizing loco-regional hypopharyngeal carcinoma recurrences in relation to FDG-PET positive and clinical radiation therapy target volumes
}

\author{
Anne K. Due, Stine Korreman, Søren M. Bentzen, Wolfgang Tomé, Edward \\ Bender, Marianne Aznar, Ivan Vogelius, Anne K. Berthelsen, Claus A. \\ Kristensen \& Lena Specht
}

To cite this article: Anne K. Due, Stine Korreman, Søren M. Bentzen, Wolfgang Tomé, Edward Bender, Marianne Aznar, Ivan Vogelius, Anne K. Berthelsen, Claus A. Kristensen \& Lena Specht (2010) Methodologies for localizing loco-regional hypopharyngeal carcinoma recurrences in relation to FDG-PET positive and clinical radiation therapy target volumes, Acta Oncologica, 49:7, 984-990, DOI: 10.3109/0284186X.2010.498833

To link to this article: https://doi.org/10.3109/0284186X.2010.498833

\section{曲 Published online: 13 Sep 2010.}

Submit your article to this journal $\pi$

Џll Article views: 758

7 Citing articles: 1 View citing articles 준 
Acta Oncologica, 2010; 49: 984-990

informa

healthcare

ORIGINAL ARTICLE

\title{
Methodologies for localizing loco-regional hypopharyngeal carcinoma recurrences in relation to FDG-PET positive and clinical radiation therapy target volumes
}

\author{
ANNE K. DUE ${ }^{1}$, STINE KORREMAN ${ }^{1,2,3}$, SØREN M. BENTZEN ${ }^{1,2}$, WOLFGANG TOMÉ ${ }^{2}$, \\ EDWARD BENDER $^{2}$, MARIANNE AZNAR ${ }^{1}$, IVAN VOGELIUS ${ }^{1}$, ANNE K. BERTHELSEN ${ }^{1}$, \\ CLAUS A. KRISTENSEN ${ }^{1}$ \& LENA SPECHT ${ }^{1}$ \\ ${ }^{1}$ Department of Radiation Oncology, Rigshospitalet, University of Copenhagen, Copenhagen, Denmark, ${ }^{2}$ Department of \\ Human Oncology and Medical Physics, University of Wisconsin, Madison, Wisconsin, USA and ${ }^{3}$ Niels Bohr Institute, \\ University of Copenhagen, Copenhagen, Denmark
}

\begin{abstract}
Background. Focal methods to determine the source of recurrence are presented, tested for reproducibility and compared to volumetric approaches with respect to the number of recurrences ascribed to the FDG-PET positive and high dose volumes. Material and methods. Six patients treated for hypopharyngeal squamous cell carcinoma were extracted from archives. Inclusion criteria were: FDG-PET/CT for primary radiotherapy planning and clinical complete remission followed by loco-regional relapse. CT scan at the time of recurrence was also required. The recurrence volume was delineated in the follow-up scans by a radiologist. Putative points of origin (PO) of the recurrence were determined by two strategies 1 ) defined by an oncologist or 2) as the center-of-volume (COV) of the recurrence. The most likely recurrence point of origin on the treatment planning scan was also determined. All expert based points of origin were repeated to estimate reproducibility. The recurrence volume and PO were propagated to the treatment planning scan using a rigid transformation. Relations of the PO to target volumes, radiation doses and therapy-points-of-origin were quantified. For the volumetric methods, the overlap of the recurrence volume and target volumes was used to determine the source of the recurrence. Results. All recurrences were located in-field, but the volumetric approaches tended to designate fewer recurrences in the PET positive volume (25\% for the 95\% threshold, 95\% confidence interval (CI):3-65\%) than the observer-based methods ( $50 \%$ for the COV and both expert evaluations on the recurrence scan, 95\% CI: 16-84\%). The reproducibility of the expert POs is better on the recurrence scan than on the therapy scan. Conclusion. Volumetric approaches favor large target volumes as the source of the recurrence, thus underestimating the number of recurrences originating in the PET positive volume. Expert based and COV approaches on the recurrence scan are the most reproducible methods to determine the PO.
\end{abstract}

Advances in radiation therapy planning and delivery technologies offer unprecedented possibilities for highly conformal irradiation of a clinical target volume (CTV). Intensity Modulated Radiation Therapy (IMRT) in particular allows dose escalation to the target volume while respecting a set of dosevolume constraints for critical normal structures in many cases. Several circumstances make this conformal therapy paradigm especially attractive and also challenging for cases with head and neck squamous cell carcinoma (HNSCC). Full clinical benefit from these technological advances requires an accurate selection and delineation of the target volume which represents a significant challenge in HNSCC. Studies have shown considerable inter-observer variability in defining clinical target volumes $[1,2]$ and there is clearly a need to improve our knowledge and to develop tools for supporting clinical target volume definition.

Three main research avenues may lead to improved definition of the target volume. 1) Patterns of failure analyses in treated patients with extended follow-up. 2) Imaging studies aimed at improved visualization of the Gross Tumor Volume (GTV) in individual cases. 
3) Probabilistic evaluation of the likelihood that a specific nodal level or anatomical compartment is involved for a given presentation of the primary.

Patterns of failure analyses have traditionally been relatively crude, in many cases just distinguishing between recurrences inside or outside the highdose CTV, with the majority of the recurrences located in-field [2-14]. An often used method is to ascribe the recurrence to the target volume encompassing at least $95 \%$ of the recurrence volume. The hypothesis in this study is that the recurrence volume is just a surrogate volume of the few cancer cells surviving radiotherapy - and we introduce a concept of the recurrence having a point of origin (PO) instead of a more regional origin, as assumed in the volume overlap method. The ability to locate this point relative to the initial target volume definition, the radiation dose distribution and the avidity of $18 \mathrm{~F}$-fluorodeoxyglucose positron emission tomography (FDG-PET) could potentially lead to rational modification of target definition and/or dose prescriptions. To this end, two types of loco-regional treatment failure are distinguished. Persistent disease refers to cases where a clinical or radiological complete remission is never achieved. Local (or loco-regional) recurrence occurs when the patient has achieved a complete response but loco-regional disease progression is subsequently seen inside or outside of the region originally thought to be involved. The present study focuses on local recurrences. Thus, the purpose of this pilot study is to develop methodologies for localization of HNSCC recurrences after radiotherapy, in relation to radiation dose, target volumes and FDG-PET positive volumes in treatment planning scans. Application of these methods in a larger cohort of HNSCC cases is in progress.

\section{Material and methods}

\section{Patients}

The background population consisted of 482 patients receiving IMRT for cancer in the head and neck area at Rigshospitalet between January 2005 and December 2008. The Picture Archiving and Communication System (PACS) of the Department of Radiology was searched on February 15, 2010 to identify patients with hypopharyngeal cancer, a radiotherapy treatment planning PET/CT and a CT at the time of recurrence. This diagnostic group was chosen since patients with hypopharyngeal carcinomas have a less favourable prognosis than other patients with squamous cell carcinomas in the head and neck area and subsequently a higher risk of relapse. Furthermore, the treatment of choice is radiotherapy without surgery. Patients should have been treated with IMRT with curative intent and achieved a clinical complete remission followed by loco-regionally failure verified by biopsy. The exclusion criteria were a previous treatment for cancer in the head and neck area, surgery prior to radiotherapy, or synchronous cancer.

\section{Primary tumour: Treatment planning and delivery}

All patients had a PET/CT scan performed for treatment planning with the integrated PET/CT Siemens Biograph 16 scanner $\left(\mathrm{CT}_{\text {therapy }}\right)$. Patients were immobilized on a flat scanner couch in the supine treatment position by an individually moulded mask covering head and shoulders.

Target volume delineation was performed in the Eclipse treatment planning system (Varian Medical System) according to local guidelines adapted from the recommendations from the Danish $\mathrm{Head}$ and Neck Cancer Group (DAHANCA) [15]. For target volume definitions, see Table I.

For hypopharyngeal squamous cell carcinoma, the total prescription radiation dose to the GTV was 66-68 Gy/33-34 fractions, 6 fractions per week. Only tumours with the largest diameter exceeding $4 \mathrm{~cm}$ were prescribed 68 Gy. The CTVE-h was prescribed a minimum dose of $60 \mathrm{~Gy}$ and the minimum dose of the CTVE-1 was 50 Gy. Daily Nimorazol and weekly Cisplatin were administered according to the DAHANCA guidelines.

IMRT plans were calculated using Eclipse. Treatments were delivered by a Varian Clinac 2300 with a dynamic 120 leaf multileaf collimator (MLC).

Table I. Target volume definitions.

\begin{tabular}{ll}
\hline Target volume & \multicolumn{1}{c}{ Definition } \\
\hline GTV-PET & $\begin{array}{c}\text { The FDG-PET positive volume, delineated } \\
\text { by a nuclear medicine physician } \\
\text { GTV }\end{array}$ \\
$\begin{array}{l}\text { The gross tumour volume, includes all } \\
\text { available diagnostic imaging as well as the } \\
\text { results of the clinical examination }\end{array}$ \\
GTV-oncologist & $\begin{array}{l}\text { The GTV with a } 1 \text { cm margin to account } \\
\text { for delination uncertainty, adjusted for } \\
\text { unaffected bone and with a } 3 \text { mm margin } \\
\text { to the skin }\end{array}$ \\
CTVE-h & $\begin{array}{l}\text { The high-risk elective clinical target volume, } \\
\text { includes areas at high risk of microscopic } \\
\text { disease. It is constructed by adding a 1 } \\
\text { cm margin to GTV-oncologist and } \\
\text { including involved lymph node levels } \\
\text { The low-risk elective clinical target volume, } \\
\text { includes areas at low but still significant } \\
\text { risk of microscopic disease. It is } \\
\text { constructed by adding a } 2 \text { mm margin to } \\
\text { CTVE-h and including lymph node levels } \\
\text { with low risk of sub clinical disease }\end{array}$ \\
\hline
\end{tabular}

The target volumes as they had been delineated on the treatment planning scans $\left(\mathrm{CT}_{\text {therapy }}\right)$. 


\section{Recurrence: Recurrence scan, delineation and} points of origin

Each patient was seen by a radiation oncologist weekly during therapy and two weeks after completion of treatment. Treatment evaluation was done two months after treatment. Hereafter, follow-up was done every four months for two years and then every six months for the following three years. Treatment failure was verified pathologically based on a biopsy or a surgical specimen. CT or PET/CT could be requested when recurrence was suspected and/or to help decide further treatment in a patient with known recurrence. The CT or PET/CT $\left(\mathrm{CT}_{\text {recur }}\right)$ at the time of recurrence was not performed in the original treatment position, but on the curved scanner couch used in the department of diagnostic radiology. After retrieving the scans performed of the patients eligible for this study, their $\mathrm{CT}_{\text {recur }}$ was transferred to Eclipse. A radiologist experienced in radiotherapy contouring delineated the recurrence volume in cooperation with a radiation oncologist with access to all available imaging information as well as the results from the clinical examination. Two different schemes were tested for identifying the point of origin (PO) within the recurrence volume. 1) An experienced radiation oncologist identified the most likely $\mathrm{PO}$ within the recurrence volume on the $\mathrm{CT}_{\text {recur }}$ and 2) the $\mathrm{PO}$ was defined as the center of volume (COV) of the manually contoured recurrence volume. For the first, observerbased, method, the identification was repeated after several days in order to test for intra-observer variability. The two points of origin were denoted PO1 and $\mathrm{PO} 2$ respectively. In addition to the definition of focal points on $\mathrm{CT}_{\text {recur }}$ the oncologist visually identified the most likely PO directly on $\mathrm{CT}_{\text {therapy }}$. In this procedure, the oncologist was blinded to the original target volumes. This definition of PO was also repeated in order to test for intra-observer variability and the resulting points were denoted $\mathrm{PO} 3$ and $\mathrm{PO} 4$ respectively.

The $\mathrm{CT}_{\text {recur }}$ was co-registered to $\mathrm{CT}_{\text {therapy }}$ using the manual, rigid co-registration tools available in Eclipse. The registration was performed to achieve the best possible match in the neck area, with spine, hyoid bone, and larynx as main landmarks. Figure 1 shows an example of a therapy scan with a PO, recurrence volume, and target volumes.

\section{Recurrence pattern analysis - the Russian Doll principle}

The defined target volumes were ordered in a hierarchy according to volume size and the chronology in which they are delineated: GTV-PET $\subseteq$ GTV $\subseteq$ GTV-oncologist $\subseteq$ CTVE-h $\subseteq$ CTVE-1. Each target volume is encompassed by the next in line. For each PO we determined the smallest target volume which

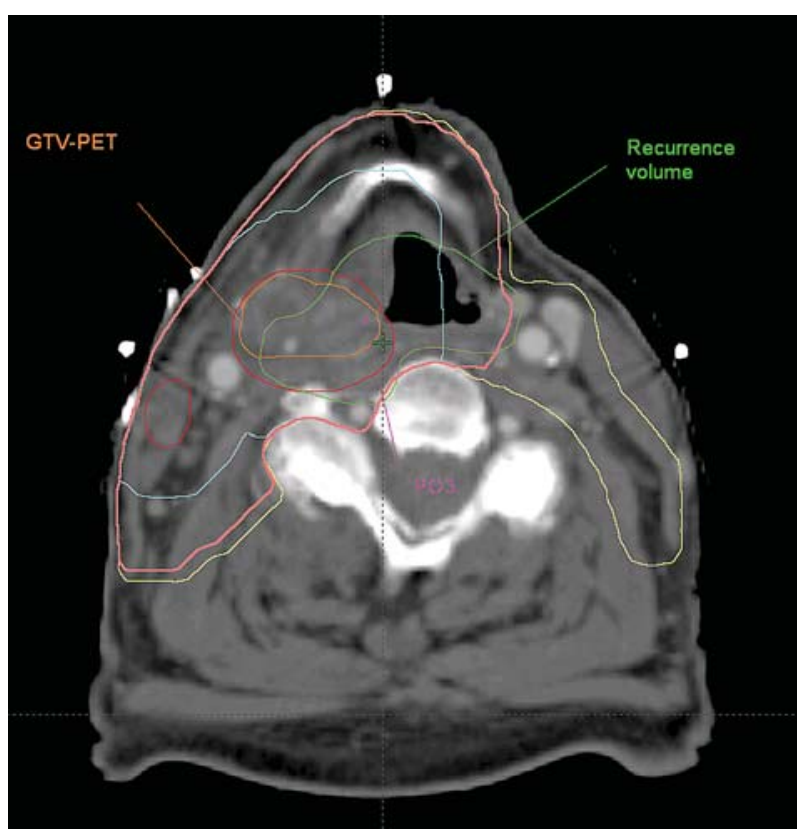

Figure 1. Treatment planning scan $\left(\mathrm{CT}_{\text {therapy }}\right)$ with the contours of the target volumes and the transferred recurrence volume and a point of origin. for this patient, all four POs and the COV were located in the GTV-PET. The recurrence volume is certainly overlapping the GTV-PET, and all the foci of the expert and COV are located therein, but the volumetric approach with a $95 \%$ threshold point to the CTVE-1 as the likely target volume where the recurrence occurs. With a $50 \%$ threshold, the volumetric approach ascribes the site of recurrence to the GTV. Magenta: Point of Origin identified on therapy scan. Orange: GTV-PET. Red: GTV. Turquoise: GTV-oncologist. Pink: CTVE-h. Yellow: CTVE-1. Green: Recurrence volume

encompassed the PO. The focal hypothesis would then imply that the recurrence originated in this target volume. A volumetric approach was also applied for comparison. With this approach we determined the smallest target volume that encompassed $50 \%$ or $95 \%$ of the recurrence volume transferred to $\mathrm{CT}_{\text {therapy }}$. The $50 \%$ and $95 \%$ threshold levels have been used in previous studies and were chosen to test whether the target volume in which a recurrence originated would depend on the threshold applied.

\section{Statistics}

Descriptive tabulations were made of the localizations of POs, COVs, and recurrence volume overlaps relative to target volumes. Exact confidence intervals were used for binomial data. Mean, range and standard deviations between POs and between POs and COVs were calculated. A Wilcoxon signed ranksum test was performed for the distances between $\mathrm{PO} 1-\mathrm{PO} 2$ and PO3-PO4.

\section{Results}

Seven patients were eligible for the study, but in one case the recurrence volume was estimated by the 
oncologist to consist of two points of origin. This rendered the COV method impossible and hence the patient was excluded. Of the remaining six patients, five had recurrence in loco and two patients had failure located in lymph nodes. The patient population is described in Table II.

The patients had target volumes delineated according to the above mentioned principles except that patient 2 did not have a CTVE-1 volume owing to the size of CTVE-h. Patient 6 had involvement of lymph nodes in level 2 and 3 on the left side and level 2 at the right side of the neck. Those were delineated, but no other lymph node levels at risk were delineated in this patient.

A total of eight relapse volumes were delineated, and eight centers of volume calculated. Furthermore, eight pairs of PO were determined on both the therapy and recurrence scan as described above. Table III shows which target volumes the different methods assigned the origin of the recurrence to. None of the eight recurrences were classified as outof-field ( $0 \%$ with $95 \%$ confidence interval (CI): $0-37 \%$ ). On $\mathrm{CT}_{\text {therapy }} 7 / 8$ points (both $\mathrm{POs}$ ) were located within the GTV-PET (88\%, with $95 \%$ CI: $47-100 \%$ ), and the most peripheral point was located in the CTVE-h.

On the $\mathrm{CT}_{\text {recur }} 4 / 8$ points (POs and COV) were located within the GTV-PET (50\% with $95 \%$ CI: $16-84 \%)$, and the most peripheral point was located in the CTVE-h.

Considering all eight recurrences and using a $50 \%$ volume threshold, four $(50 \%$ with $95 \%$ CI: 16-84\%) recurrences were located within the GTVoncologist and three (38\% with 95\% CI: 9-76\%) and one $(13 \%$ with $95 \%$ CI: $0-53 \%)$ in the GTVPET and the GTV, respectively. Using a 95\% volume threshold, three (38\% with 95\% CI: 9-76\%) recurrences were located within the GTV-oncologist, but two (25\% with $95 \%$ CI: 3-65\%) were located even more peripherally, outside of the high dose volume. Two recurrences occurred in the GTV-PET (25\% with 95\% CI: 3-65\%) and one in the GTV.

Table III displays all 56 assignments of the target volume where the recurrence originated. Fifty-two of the volumes received $66-68$ Gy, three received 60 Gy and one volume $50 \mathrm{~Gy}$.

Figure 2 shows a plot of the mean and range of the distances between the two POs on each scan and the distance between the COV method and the POs on the recurrence scan. On $\mathrm{CT}_{\text {recur }}$ the mean distance between $\mathrm{PO} 1$ and $\mathrm{PO} 2$ is $5 \mathrm{~mm}$ (range $0-9$ $\mathrm{mm}$, SD $3 \mathrm{~mm}$ ). On $\mathrm{CT}_{\text {therapy }}$ the mean distance between PO3 and PO4 is $10 \mathrm{~mm}$ (range $3-26 \mathrm{~mm}$, $\mathrm{SD} 7 \mathrm{~mm}$ ). A Wilcoxon signed rank-sum test for difference between the PO1-PO2 and PO3-PO4 pairs of distances is non-significant $(\mathrm{p}=0.11)$ but the statistical power is low due to the small sample size. The mean distances between $\mathrm{PO} 1 / \mathrm{PO} 2$ and $\mathrm{COV}$ are 4 $\mathrm{mm}$ (range $1-8 \mathrm{~mm}$, SD $3 \mathrm{~mm}$ ) and $7 \mathrm{~mm}$ (range 1-10 $\mathrm{mm}$, SD $3 \mathrm{~mm}$ ) respectively.

\section{Discussion}

The two conceptually different methods of defining the target volume encompassing the source of recurrence, the focal and volumetric approach, give differing results. The focal methods, whether expert based or based on COV, and on both the $\mathrm{CT}_{\text {recur }}$ and the $\mathrm{CT}_{\text {therapy }}$ tend to define more recurrences as originating in the central structures than the volumetric method. This can be understood from Figure 1. In this patient, all observer based foci are placed in GTV-PET in accordance with the COV focus (not shown). In contrast the $50 \%$ volumetric method designates GTV-oncologist as the source, whereas the $95 \%$ volumetric approach designates the CTVE1 as the source. The cause of this discrepancy is that the volumetric approach favours large target volumes. If a target volume is smaller than $95 \%$ of the recurrence volume, it can never be considered the source of the recurrence using the volumetric definition with a $95 \%$ threshold. Hence attribution of the recurrence to a small target volume becomes less likely as the recurrence volume increases. The target volume designated as the source of the recurrence with the $95 \%$ thresholds will always be the same or a larger target volume than the target volume designated as the source with the $50 \%$ threshold. This is

Table II. Patient characteristics.

\begin{tabular}{llclllll}
\hline Patient & Gender & Age & TNM & \multicolumn{1}{c}{ RT } & Concomitant medication & Tumour localization & Site of recurrence \\
\hline 1 & male & 63 & T2 N2b M0 & $2 \mathrm{~Gy} \times 33,6 \mathrm{~F} / \mathrm{w}$ & Nimorazol & Left pyriform sinus & $\mathrm{T}$ \\
2 & male & 62 & T3 N2c M0 & $2 \mathrm{~Gy} \times 34,6 \mathrm{~F} / \mathrm{w}$ & Nimorazol Cisplatin & Right pyriform sinus & $\mathrm{T}$ and $2 \mathrm{~N}$ \\
3 & male & 70 & T3 N2a Mx & $2 \mathrm{~Gy} \times 34,6 \mathrm{~F} / \mathrm{w}$ & Nimorazol & Right pyriform sinus & $\mathrm{T}$ \\
4 & female & 67 & T2 N0 M0 & $2 \mathrm{~Gy} \times 33,6 \mathrm{~F} / \mathrm{w}$ & Nimorazol & Posterior hypopharyngeal wall & $\mathrm{T}$ \\
5 & male & 71 & T2 N0 M0 & $2 \mathrm{~Gy} \times 33,6 \mathrm{~F} / \mathrm{w}$ & Nimorazol & Right pyriform sinus & $\mathrm{T}$ \\
6 & male & 71 & T3 N2b M1 & $2 \mathrm{~Gy} \times 34,6 \mathrm{~F} / \mathrm{w}$ & Nimorazol Cisplatin & Left pyriform sinus & $\mathrm{N}$ \\
\hline
\end{tabular}

Demographical data, treatment related data and location of recurrence for the six patients included in the study.

TNM: tumour, node, metastasis classification; $F / w$ : fractions per week. 
Table III. Recurrences and target volumes.

\begin{tabular}{|c|c|c|c|c|c|c|}
\hline \multirow[b]{2}{*}{ Scan } & \multirow[b]{2}{*}{ Method } & \multicolumn{5}{|c|}{ Volume } \\
\hline & & GTV-PET & GTV & GTV-oncologist & CTVE-h & CTVE-1 \\
\hline \multirow[t]{3}{*}{ Recurrence CT } & Expert PO1 & $4 / 8$ & $1 / 8$ & $3 / 8$ & $0 / 8$ & $0 / 8$ \\
\hline & Expert PO2 & $4 / 8$ & $2 / 8$ & $1 / 8$ & $1 / 8$ & $0 / 8$ \\
\hline & Centre of volume & $4 / 8$ & $2 / 8$ & $2 / 8$ & $0 / 8$ & $0 / 8$ \\
\hline Treatment & Expert PO3 & $7 / 8$ & $0 / 8$ & $1 / 8$ & $0 / 8$ & $0 / 8$ \\
\hline Planning CT & Expert PO4 & $7 / 8$ & $0 / 8$ & $0 / 8$ & $1 / 8$ & $0 / 8$ \\
\hline \multicolumn{7}{|l|}{ Volume overlaps } \\
\hline $50 \%$ threshold & & $3 / 8$ & $1 / 8$ & $4 / 8$ & $0 / 8$ & $0 / 8$ \\
\hline $95 \%$ threshold & & $2 / 8$ & $1 / 8$ & $3 / 8$ & $1 / 8$ & $1 / 8$ \\
\hline Prescription dose & & 66-68 Gy & $66-68 \mathrm{~Gy}$ & 66-68 Gy & $60 \mathrm{~Gy}$ & $50 \mathrm{~Gy}$ \\
\hline
\end{tabular}

Table III. Location of points of origin in target volumes as estimated by the expert on the recurrence scan and the treatment planning scan as well as the location estimated with the centre of volume method and the volumetric overlap method. The dose prescribed to the target volume is indicated in the bottom line of the table.

PO1 and PO2: Double determinants of the expert defined point of origin within the recurrence volume.

PO3 and PO4: Double determinants of the expert defined point of origin on the treatment planning CT.

confirmed in Table III, where the 95\% threshold generally locates the source of the recurrence in larger volumes than the other volumetric approach as well as the focal methods. This means, that the volumetric criterion will tend to underestimate the potential benefit from a central radiation dose boost as compared to the focal method. In contrast, the COV approach will define the same source of recurrence, independent of time and growth rate to the extent that the growth of the recurrence is symmetrical. Other studies have shown that the majority of the recurrences occur in-field which is consistent with our result. The pattern of failure analyses in these studies have been performed using either the volume overlap method or an expert identifying the

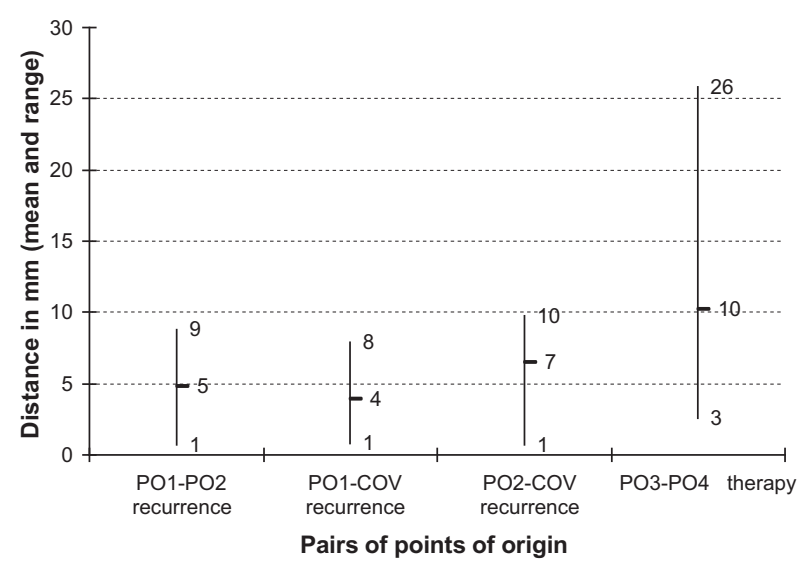

Figure 2. Distances between the repeated expert evaluated PO on the recurrence and therapy scans and the distance between the $\mathrm{COV}$ of the recurrence and the expert evaluated POs on the recurrence scan. A trend is seen towards the consistency between expert evaluations being better in the recurrence scan than in the therapy scan. The COV method on the recurrence scan appears to be as consistent with the expert evaluated POs on two consecutive expert evaluations. most likely site of recurrence origin directly on the treatment planning scan. The perspective of this study is to develop a method that enables a more accurate location of the recurrence in relation to the smaller sub-volumes within the high-dose region.

A higher reproducibility was observed for POs defined on the $\mathrm{CT}_{\text {recur }}$ than on the $\mathrm{CT}_{\text {therapy }}$ (Figure 2). A possible explanation could be that the POs on the $\mathrm{CT}_{\text {recur }}$ are defined within a small volume whereas the POs on the $\mathrm{CT}_{\text {therapy }}$ were defined on a scan with no pre-defined volumes. We hypothesized that the center of volume approach could potentially be more robust than a subjectively chosen single point of origin defined by an expert. At present, we can only conclude that the mean distance between $\mathrm{PO} 1 / \mathrm{PO} 2$ and $\mathrm{COV}$ are comparable to the reproducibility between the double-determined points $\mathrm{PO} 1$ and $\mathrm{PO} 2$, suggesting that the two methods may be equivalent. A limitation of the COV method is the cases where a recurrence volume contains more POs. In such cases, the COV method cannot be used.

Multiple steps are required for each of the procedures for localizing the recurrence point of origin on the therapy scan, each associated with several inaccuracies. Delineation of recurrence volume is difficult as the relapse will often occur in-field, i.e. in a heavily irradiated anatomical region with subsequent post-treatment changes [16]. Delineation intra- and inter-observer variation is presumably even larger than in delineation of the primary tumour, although systematic studies of this have not been conducted to the best of our knowledge. Transferring the recurrence volume and/or PO from $\mathrm{CT}_{\text {recur }}$ to $\mathrm{CT}_{\text {therapy }}$ is a crucial step irrespective of method used for locating the PO. Various strategies have been tested by other groups [3-14,17]. Image 
co-registration can be performed using rigid and deformable algorithms $[4-6,8,10-12,17]$ In this study we used manual rigid co-registration for registration of the PET/CT $T_{\text {therapy }}$ and $\mathrm{CT}_{\text {recur }}$. Rigid coregistration with translations and rotations is relatively easy to use but is not satisfactory in the presence of large variations in patient anatomy, weight and position. Deformable co-registration can to some extent account for this variation, but introduces the challenge of assuring an anatomically plausible vector field. A significant problem with co-registration of $\mathrm{CT}_{\text {recur }}$ and $\mathrm{CT}_{\text {therapy }}$ is the lack of topological equivalence in the area of the cancer, as the primary tumor is present on $\mathrm{CT}_{\text {therapy }}$ but not on $\mathrm{CT}_{\text {recur }}$ - and the recurrence volume vice versa. Transferring points and volumes by co-registration of scans involves assumptions and approximations and an expert opinion might be just as good. Some studies have used an expert approach - to transfer the recurrence volume or simply to determine where within the target volume the recurrence occurred $[3,7,9,13,14]$. We used a similar method by letting the radiation oncologist determine a point on $\mathrm{CT}_{\text {therapy }}$ that was estimated to be the most likely origin of the recurrence. Seven out of eight points were located in GTV-PET, but the points showed a lower reproducibility compared to the POs on the $\mathrm{CT}_{\text {recur }}$.

None of the loco-regional failures were geographical misses, but with the current sample size it cannot be decided meaningfully whether the defined target volumes were too liberal. However, based on this small pilot series, treatment intensity seems to be the success-limiting factor. As current non-surgical management of these tumours may be close to patient tolerance $[18,19]$, further increase of the locoregional treatment intensity may require novel approaches such as dose painting [20-25]. The benefit of boosting the dose to the central target volumes may be underestimated using the volume overlap method, whereas the focal method lends some support for dose-painting strategies boosting the dose to FDG-avid regions.

\section{Conclusion}

Localizing loco-regional recurrences after definitive radiotherapy for hypopharyngeal carcinoma using the overlap of the recurrence volume with target volumes tended to place the recurrence in more peripheral target volumes, whereas the focal method placed the point of origin in more central target volumes. Among the focal methods, a center of volume approach based on delineation of the recurrence volume seem to perform as well as an expert defined point of origin on the recurrence CT scan.

\section{Acknowledgements}

Supported by CIRRO - The Lundbeck Foundation Center for Interventional Research in Radiation Oncology, University of Copenhagen and the Danish Graduate School for Clinical Oncology. SMB acknowledges support from the National Cancer Institute grant no. 2P30 CA 014520-34.

Declaration of interest: The authors report no conflicts of interest. The authors alone are responsible for the content and writing of the paper.

\section{References}

[1] Breen SL, Publicover J, De SS, Pond G, Brock K, O'Sullivan $B$, et al. Intraobserver and interobserver variability in GTV delineation on FDG-PET-CT images of head and neck cancers. Int J Radiat Oncol Biol Phys 2007;68:763-70.

[2] Chao KS, Bhide S, Chen H, Asper J, Bush S, Franklin G, et al. Reduce in variation and improve efficiency of target volume delineation by a computer-assisted system using a deformable image registration approach. Int J Radiat Oncol Biol Phys 2007;68:1512-21.

[3] Bussels B, Maes A, Hermans R, Nuyts S, Weltens C, Van den $\mathrm{BW}$. Recurrences after conformal parotid-sparing radiotherapy for head and neck cancer. Radiother Oncol 2004; 72:119-27.

[4] Caudell JJ, Meredith RF, Spencer SA, Keene KS, Dobelbower MC, Bonner JA. Margin on gross tumor volume and risk of local recurrence in head-and-neck cancer. Int J Radiat Oncol Biol Phys 2010;76:164-8.

[5] Daly ME, Lieskovsky Y, Pawlicki T, Yau J, Pinto H, Kaplan $M$, et al. Evaluation of patterns of failure and subjective salivary function in patients treated with intensity modulated radiotherapy for head and neck squamous cell carcinoma. Head Neck 2007;29:211-20.

[6] Daly ME, Le QT, Maxim PG, Loo BW, Jr., Kaplan MJ, Fischbein NJ, et al. Intensity-modulated radiotherapy in the treatment of oropharyngeal cancer: Clinical outcomes and patterns of failure. Int J Radiat Oncol Biol Phys 2010; 76:1339-46.

[7] Dawson LA, Anzai Y, Marsh L, Martel MK, Paulino A, Ship JA, et al. Patterns of local-regional recurrence following parotid-sparing conformal and segmental intensitymodulated radiotherapy for head and neck cancer. Int J Radiat Oncol Biol Phys 2000;46:1117-26.

[8] Eisbruch A, Marsh LH, Dawson LA, Bradford CR, Teknos TN, Chepeha DB, et al. Recurrences near base of skull after IMRT for head-and-neck cancer: Implications for target delineation in high neck and for parotid gland sparing. Int J Radiat Oncol Biol Phys 2004;59:28-42.

[9] Lee N, Xia P, Fischbein NJ, Akazawa P, Akazawa C, Quivey JM. Intensity-modulated radiation therapy for head-and-neck cancer: The UCSF experience focusing on target volume delineation. Int J Radiat Oncol Biol Phys 2003;57:49-60.

[10] Sanguineti G, Gunn GB, Endres EJ, Chaljub G, Cheruvu P, Parker B. Patterns of locoregional failure after exclusive IMRT for oropharyngeal carcinoma. Int J Radiat Oncol Biol Phys 2008;72:737-46.

[11] Schoenfeld GO, Amdur RJ, Morris CG, Li JG, Hinerman RW, Mendenhall WM. Patterns of failure and toxicity after intensity-modulated radiotherapy for head and neck cancer. Int J Radiat Oncol Biol Phys 2008;71:377-85. 
[12] Soto DE, Kessler ML, Piert M, Eisbruch A. Correlation between pretreatment FDG-PET biological target volume and anatomical location of failure after radiation therapy for head and neck cancers. Radiother Oncol 2008;89:13-8.

[13] Studer G, Luetolf UM, Glanzmann C. Locoregional failure analysis in head-and-neck cancer patients treated with IMRT. Strahlenther Onkol 2007;183:417-23.

[14] Yao M, Dornfeld KJ, Buatti JM, Skwarchuk M, Tan H, Nguyen $\mathrm{T}$, et al. Intensity-modulated radiation treatment for head-and-neck squamous cell carcinoma-the University of Iowa experience. Int J Radiat Oncol Biol Phys 2005;63: 410-21.

[15] DAHANCA guidelines. Available from: http://www.dahanca. $\mathrm{dk} /$ guidelines. May 1, 2010.

[16] Hermans R. Posttreatment imaging in head and neck cancer. Eur J Radiol 2008;66:501-11.

[17] Chao KSC, Ozyigit G, Tran BN, Cengiz M, Dempsey JF, Low DA. Patterns of failure in patients receiving definitive and postoperative IMRT for head-and-neck cancer. Int J Radiat Oncol Biol Phys 2003;55:312-21.

[18] Bentzen SM, Rosenthal DI, Weymuller EA, Trotti A. Increasing toxicity in nonoperative head and neck cancer treatment: Investigations and interventions. Int $\mathrm{J}$ Radiat Oncol Biol Phys 2007;69:S79-S82.
[19] Bentzen SM, Trotti A. Evaluation of early and late toxicities in chemoradiation trials. J Clin Oncol 2007;25:4096-103.

[20] Assenholt MS, Petersen JB, Nielsen SK, Lindegaard JC, Tanderup K. A dose planning study on applicator guided stereotactic IMRT boost in combination with 3D MRI based brachytherapy in locally advanced cervical cancer. Acta Oncol 2008;47:1337-43.

[21] Bentzen SM. Theragnostic imaging for radiation oncology: Dose-painting by numbers. Lancet Oncol 2005;6:112-7.

[22] Christian N, Lee JA, Bol A, De BM, Jordan B, Gregoire V. The limitation of PET imaging for biological adaptive-IMRT assessed in animal models. Radiother Oncol 2009;91:101-6.

[23] KimY, Tome WA. Is it beneficial to selectively boost high-risk tumor subvolumes? A comparison of selectively boosting high-risk tumor subvolumes versus homogeneous dose escalation of the entire tumor based on equivalent EUD plans. Acta Oncol 2008;47:906-16.

[24] Ling CC, Humm J, Larson S, Amols H, Fuks Z, Leibel S, Koutcher JA. Towards multidimensional radiotherapy (MD-CRT): Biological imaging and biological conformality. Int J Radiat Oncol Biol Phys 2000;47:551-60.

[25] Toma-Dasu I, Dasu A, Brahme A. Dose prescription and optimisation based on tumour hypoxia. Acta Oncol 2009; 48:1181-92. 